\title{
PREVALÊNCIA DE SOBREPESO E OBESIDADE EM CRIANÇAS DE DOIS A SEIS ANOS MATRICULADAS EM ESCOLAS PARTICULARES NO MUNICÍPIO DE SÃO PAULO
}

\section{PREVALENCE OF OVERWEIGHT AND OBESITY IN TWO TO SIX YEAR OLD CHILDREN REGISTERED IN PRIVATE SCHOOLS IN SÃO PAULO}

Viviane Gabriela Nascimento Simon ${ }^{1}$

José Maria Pacheco de Souza ${ }^{2}$

Claudio Leone ${ }^{3}$

Sonia Buongermino de Souza ${ }^{4}$

Simon VGN et al. Prevalência de sobrepeso e obesidade em crianças de dois a seis anos matriculadas em escolas particulares no município de São Paulo. Rev Bras Crescimento Desenvolv Hum. 2009; 19(2):211-218.

\section{Resumo:}

Objetivo: verificar a prevalência de sobrepeso e obesidade segundo sexo e idade em crianças de 2 a 6 anos de idade, alunos de escolas particulares no município de São Paulo. Método: foram realizadas medidas de peso e de altura para verificação do estado nutricional de oitocentos e seis crianças de ambos os sexos. Para a classificação do estado nutricional das crianças foram utilizadas as curvas de percentis do Índice de Massa Corporal (IMC = Peso $(\mathrm{kg}) /$ Altura $\left.^{2}(\mathrm{~cm})\right)$ para idade, conforme padrão de referência do Multicentre Growth Study, recomendado pela Organização Mundial de Saúde que classifica como sobrepeso valores de percentis $\geq 85$ e $<97$ e para a obesidade valores $<97$. Para análise da relação entre sexo, idade da criança e estado nutricional utilizou-se modelo linear generalizado de regressão múltipla (glm) com ligação logarítmica e família binomial, que permite, diretamente, a estimação das razões de prevalências. A prevalência de sobrepeso+obesidade foi $37,2 \%$ para o sexo masculino e $33,4 \%$ para o sexo feminino. A razão de prevalência (RP) mostrou que não existe diferença significativa entre obesidade e sobrepeso+obesidade para sexo e idade. Conclusão: observaram-se prevalências de sobrepeso e de obesidade superiores às prevalências médias da população brasileira. Os resultados encontrados neste estudo reforçam a preocupação com a obesidade infantil que aparentemente vem crescendo, em idades mais precoces como dos pré-escolares.

Plavras-chave: prevalência; sobrepeso; obesidade; pré-escolar.

Pós-Doutoranda do Departamento de Saúde Materno Infantil da Faculdade de Saúde Pública da Universidade de São Paulo Professor Titular do Departamento de Epidemiologia da Faculdade de Saúde Pública da Universidade de São Paulo Professor Titular do Departamento de Saúde Materno-Infantil da Faculdade de Saúde Pública da Universidade de são Paulo. Professora Doutora do Departamento de Nutrição da Faculdade de Saúde Pública da Universidade de São Paulo.

Artigo baseado em pesquisa elaborada para tese de doutorado de VGN Simon, apresentada à Faculdade de Saúde Pública da Universidade de São Paulo, em 2007, intitulada "Relação entre aleitamento materno, alimentação complementar e obesidade e sobrepeso em crianças de 2 a 6 anos de idade matriculadas em escolas particulares no Município de São Paulo".

Faculdade de Saúde Pública da Universidade de São Paulo - Av. Dr. Arnaldo, 715 - CEP 01246-904. São Paulo, SP - Brasil. - Email para contato: vivianesimon@usp.br 


\begin{abstract}
:
to verify the prevalence of overweight and obesity according to gender and age in two to six years old children registered in private schools of São Paulo. Methods: the measured of weight and height in order to assess the nutritional state of eight hundred and six children from both sexes. In order to perform the classification of the nutritional state of children we used the percentiles curves of Body Mass Index $\left(B M I=\right.$ Weight $(\mathrm{kg}) /$ Height $\left.(\mathrm{cm})^{2}\right)$ for age as reference standard of Multicentre Growth Study, currently recommended for the World Health Organization that classifies as overweight the percentiles values e" 85 to $<97$, and for obesity the values $>97$. In order to analyze the relation between sex, age and nutritional status we used the linear model generalized of multiple regressions (glm) with logarithmic relating, and binomial family, which provides, directly, the value of the ratios of prevalence (RP). Results: The prevalence of overweight + obesity was $37.2 \%$ for male and $33.4 \%$ for female children. The prevalence ratios (PR) showed that a significant difference between obesity and overweight + obesity for sex and age does not exist. Conclusion: Prevalence of overweight and obesity superior than the average prevalence of the Brazilian population were observed. The results found in this study strengthen the concern with the infantile obesity that apparently is increasingly growing in precocious ages as of the preschool ones children.
\end{abstract}

Key word: prevalence; overweight; obesity; child and preschool.

\section{INTRODUÇÃO}

A obesidade é "uma síndrome multifatorial que consiste em alterações fisiológicas, bioquímicas, metabólicas, anatômicas, além de alterações psicológicas e sociais, sendo caracterizada pelo aumento de adiposidade e de peso corporal" ", envolvendo fatores genéticos e ambientais.$^{2}$ Há evidências de que fatores genéticos são capazes de modular a resposta do organismo às variações dos fatores ambientais, como dieta e atividade física. ${ }^{3} \mathrm{~A}$ ingestão de dieta com valor calórico elevado e o gasto energético diminuído têm sido apontados como os principais fatores causais da obesidade. ${ }^{4,5}$

Em relação à obesidade infantil, Lustig ${ }^{6} \mathrm{a}$ descreve como um fenômeno associado a diversas doenças, a maioria das quais ainda não totalmente identificadas. É consenso que a obesidade infantil vem aumentando de forma significativa e que determina vários agravos à saúde na infância e na idade adulta. ${ }^{7}$

Atualmente, dentre os problemas que acometem as crianças, além das formas crônicas e agudas de desnutrição moderada e grave, o sobrepeso vem sendo uma importante questão enfrentada nos Sistemas de Vigilância Alimentar e Nutricional. ${ }^{8}$

Em alguns paises da América Latina, que apresentaram rápido crescimento econômico nas últimas décadas, observou-se tendência temporal de diminuição da desnutrição infantil associada ao aumento da prevalência da obesidade $^{9}$; o mesmo tem sido verificado no Brasi $^{10} \mathrm{e}$, fato que tem preocupado os especialistas, o aparecimento do sobrepeso e da obesidade tem sido constatado em idades cada vez mais precoces. ${ }^{11-13}$

Estudos realizados em algumas cidades brasileiras mostram que a prevalência, tanto do sobrepeso quanto da obesidade é maior na população mais favorecida economicamente ${ }^{14-17}$, ao contrário do que ocorre nos paises desenvolvidos, onde a maioria das crianças com sobrepeso ou obesas pertencem a famílias de condição socioeconômica baixa. ${ }^{18}$

Os dados da Pesquisa Nacional sobre Saúde e Nutrição (PNSN) de 1989 já mostravam 
que, no Brasil, havia cerca de um milhão de crianças com sobrepeso, e a prevalência era maior entre meninas do que em meninos, sendo que, nas regiões Sul e Sudeste, as prevalências de sobrepeso se aproximavam do dobro da encontrada no Nordeste, e as regiões Norte e Centro-Oeste se encontravam em situação intermediária. Nas famílias pertencentes à faixa superior de renda, a proporção de crianças nessa condição era em torno de $8,0 \%$, enquanto que em famílias de renda inferior essa taxa era de $2,5 \%$. A prevalência de sobrepeso predominava nos dois primeiros anos de vida. ${ }^{19}$

Dados preliminares da Pesquisa Nacional de Demografia e Saúde (PNDS) de 2006 mostram que $7 \%$ das crianças brasileiras menores de cinco anos estão em situação de excesso de peso, variando de $6 \%$ na região Norte a $9 \%$ na região Sul, indicando exposição moderada à obesidade em todas as regiões do país. ${ }^{20}$

Segundo Monteiro et al (2000) $)^{21}$, no Brasil, nos últimos 30 anos, o número de crianças com peso acima do normal triplicou, enquanto nos Estados Unidos duplicou. Esse aumento foi mais importante nas regiões Sul e Sudeste do Brasil, nas camadas mais favorecidas da população.

O excesso de peso já é um desvio nutricional relevante por sua intensidade e freqüência, portanto, o objetivo deste estudo foi verificar a prevalência de sobrepeso e obesidade segundo sexo e idade em crianças de dois a seis anos, alunos de escolas particulares no Município de São Paulo.

\section{MÉTODO}

Trata-se de corte transversal, com crianças de 2 a 6 anos de idade completos, matriculadas em escolas particulares, com valor de mensalidade de dois salários mínimos ou mais que, em sua maioria, permite supor que essas crianças pertencem a famílias de melhor nível socioeconômico.
Este estudo utiliza informações do banco completo de uma pesquisa intitulada "Relação do aleitamento materno, alimentação complementar e obesidade e sobrepeso em crianças de 2 a 6 anos de idade matriculadas em escolas particulares no Município de São Paulo". Naquela pesquisa, a amostra foi calculada por meio dos seguintes parâmetros: nível de significância do teste $\mu=5 \%$; poder do teste (1b) $=80 \%$; odds ratio $=2,2$; relação de $3: 1$ entre obesos e não obesos; \% de expostos entre não obesos de $11 \%$. Para factibilidade e maior operacionalidade no desenvolvimento dessa pesquisa, foi realizado contato telefônico com sete escolas particulares, localizadas no bairro de Santana, na Zona Norte do município de São Paulo, que concordaram em participar do estudo.

Foram excluídas do estudo crianças que apresentaram doenças crônicas ou específicas do crescimento, que não compareceram nas creches nos dias marcados para coleta de dados, e as crianças cujos pais não autorizaram a participação.

Houve consentimento por parte dos pais para que todas as crianças das sete escolas, com idade entre 2 e 6 anos, participassem do estudo, não havendo exclusão de crianças, e a amostra total foi de 806 crianças e para este estudo essa amostra é usada integralmente.

O período de coleta de dados foi de agosto a novembro de 2004 e de março a maio de 2005. Data de nascimento, idade atual, sexo, peso e altura atuais das crianças foram coletados em formulário específico deste estudo.

Para coleta dos dados antropométricos, foi realizada medida de peso com balança Tanita Solar Scale 1632, e de estatura com o estadiômetro Seca Bodymeter 208. Todas as medidas antropométricas foram obtidas utilizando método descrito por Lohman et al..$^{22} \mathrm{Os}$ dados antropométricos das crianças foram coletados na própria escola.

As variáveis sexo e idade das crianças (variável contínua, em anos inteiros) foram con- 
sideradas variáveis explanatórias. A variável, dependente do estudo foi estado nutricional da criança. Para sua classificação, foram utilizadas as curvas de percentis do Índice de Massa Corporal $\left(\mathrm{IMC}=\right.$ Peso $(\mathrm{kg}) /$ Altura $\left.(\mathrm{cm})^{2}\right)$ para idade, conforme padrão de referência o Multicentre Growth Study, atualmente recomendado pela Organização Mundial de Saú$\mathrm{de}^{23}$ que classifica como baixo IMC para idade valores $<$ percentil 3 , IMC adequado ou eutrófico $>$ percentil $3 \mathrm{e}<$ percentil 85 , sobrepeso $>$ percentil $85 \mathrm{e}<$ percentil 97 e para obesidade valores $>$ percentil 97 . Para análise estatística, a variável dependente foi categorizada em baixo IMC para idade + IMC adequado ou eutrófico ( $<$ percentil 85) e sobrepeso + obesidade $(>$ percentil 85$)$.

Para a descrição dos resultados, foram utilizadas freqüências, porcentagens, medidas centrais e de dispersão. Para análise da relação entre as variáveis explanatórias (sexo e idade da criança) e a variável dependente (estado nutricional da criança), utilizou-se regressão linear generalizada múltipla (glm) com ligação logarítmica e família binomial, que permite diretamente a estimação das razões de prevalências (RP). ${ }^{24} \mathrm{O}$ uso da modelagem me- diante glm inclui teste de hipóteses, que pode ser feito usando o valor de $\mathrm{p}$ descritivo correspondente à razão de prevalência (RP) de interesse. Adotou-se nível de 5\% de significância. $\mathrm{Na}$ análise, foram considerados dois grupos: obesidade e sobrepeso + obesidade segundo sexo e idade.

O banco de dados foi elaborado utilizandose a planilha Microsoft Excel 2002 e as análises estatísticas foram feitas utilizando-se o pacote Stata 10.1. Todas as mães e/ou responsáveis pelas crianças concordaram com a participação na pesquisa e assinaram um Termo de Consentimento Livre e Esclarecido. O presente estudo foi aprovado pelo Comitê de Ética em Pesquisa da Faculdade de Saúde Pública da Universidade de São Paulo (Protocolo $\mathrm{n}^{\mathrm{o}} 1222$, de fevereiro de 2005).

\section{RESULTADOS}

Observou-se prevalência de excesso de peso de $35,4 \%$ na amostra como um todo $(n=806)$, mostrando que as prevalências de excesso de peso não diferem segundo sexo e idade das crianças.

Tabela 1: Distribuição da amostra de pré-escolares, segundo idade, sexo e estado nutricional. São Paulo, SP, 2004/2005.

\begin{tabular}{|c|c|c|c|c|c|c|c|c|c|c|}
\hline \multirow{2}{*}{$\begin{array}{l}\text { Idade } \\
\text { (anos) }\end{array}$} & \multicolumn{2}{|c|}{$\begin{array}{l}\text { Baixo IMC } \\
\text { para idade }\end{array}$} & \multicolumn{2}{|c|}{$\begin{array}{l}\text { IMC adequado } \\
\text { ou Eutrófico }\end{array}$} & \multicolumn{2}{|c|}{ Sobrepeso } & \multicolumn{2}{|c|}{ Obesidade } & \multicolumn{2}{|c|}{ Total } \\
\hline & $\mathbf{n}^{\circ}$ & $\%$ & $\mathbf{n}^{\circ}$ & $\%$ & $\mathbf{n}^{\circ}$ & $\%$ & $n^{\circ}$ & $\%$ & $n^{\circ}$ & $\%$ \\
\hline \multicolumn{11}{|c|}{ SEXO MASCULINO } \\
\hline 2 & - & - & 30 & 55,5 & 13 & 24,1 & 11 & 20,4 & 54 & 100 \\
\hline 3 & - & - & 53 & 68,8 & 17 & 22,1 & 7 & 9,1 & 77 & 100 \\
\hline 4 & 1 & 0,9 & 62 & 59,6 & 25 & 24,0 & 16 & 15,4 & 104 & 100 \\
\hline 5 & 1 & 1,1 & 60 & 63,8 & 14 & 14,9 & 19 & 20,2 & 94 & 100 \\
\hline 6 & - & - & 49 & 66,2 & 16 & 20,2 & 14 & 17,7 & 79 & 100 \\
\hline Total & 2 & 0,5 & 254 & 62,2 & 85 & 20,8 & 67 & 16,4 & 408 & 100 \\
\hline \multicolumn{11}{|c|}{ SEXO FEMININO } \\
\hline 2 & - & - & 39 & 61,9 & 14 & 22,2 & 10 & 15,9 & 63 & 100 \\
\hline 3 & 1 & 1,3 & 53 & 68,8 & 13 & 16,9 & 10 & 13,0 & 77 & 100 \\
\hline 4 & - & - & 71 & 69,6 & 24 & 23,5 & 7 & 6,9 & 102 & 100 \\
\hline 5 & - & - & 63 & 64,9 & 21 & 21,6 & 13 & 13,4 & 97 & 100 \\
\hline 6 & - & - & 38 & 64,4 & 12 & 20,3 & 9 & 15,2 & 59 & 100 \\
\hline Total & 1 & 0,2 & 264 & 66,3 & 84 & 21,1 & 49 & 12,3 & 398 & 100 \\
\hline
\end{tabular}


Tabela 2: Características antropométricas de pré-escolares, de acordo com idade e sexo. São Paulo, SP, 2004/2005.

\begin{tabular}{|c|c|c|c|c|c|c|}
\hline \multirow{2}{*}{$\begin{array}{l}\text { Idade } \\
\text { (anos) }\end{array}$} & \multicolumn{2}{|c|}{ Peso (kg) } & \multicolumn{2}{|c|}{ Altura (m) } & \multicolumn{2}{|c|}{ IMC $\left(\mathrm{kg} / \mathrm{m}^{2}\right)$} \\
\hline & Média & dp & Média & dp & Média & dp \\
\hline \multicolumn{7}{|c|}{ SEXO MASCULINO } \\
\hline 2 & 14,70 & 2,68 & 0,92 & 0,06 & 17,4 & 2,29 \\
\hline 3 & 16,36 & 2,31 & 1,00 & 0,06 & 16,24 & 1,62 \\
\hline 4 & 19,59 & 3,15 & 1,09 & 0,05 & 16,46 & 1,81 \\
\hline 5 & 22,67 & 4,70 & 1,16 & 0,05 & 16,71 & 2,82 \\
\hline 6 & 24,48 & 5,11 & 1,20 & 0,05 & 16,92 & 2,61 \\
\hline \multicolumn{7}{|c|}{ SEXO FEMININO } \\
\hline 2 & 14,22 & 2,48 & 0,91 & 0,05 & 16,90 & 1,93 \\
\hline 3 & 16,08 & 2,75 & 1,00 & 0,05 & 16,25 & 1,86 \\
\hline 4 & 18,64 & 2,77 & 1,07 & 0,04 & 16,25 & 1,82 \\
\hline 5 & 22,12 & 3,93 & 1,15 & 0,06 & 16,56 & 2,01 \\
\hline 6 & 24,12 & 4,87 & 1,20 & 0,05 & 16,57 & 2,41 \\
\hline
\end{tabular}

Figura 1: Proporções de sobrepeso, obesidade e sobrepeso + obesidade, segundo idade em anos completos, São Paulo, SP, 2004/2005.



Tabela 3: Análise da obesidade e sobrepeso + obesidade, segundo o modelo linear generalizado de regressão múltipla. São Paulo, SP, 2004/2005.

\begin{tabular}{lccc}
\hline Variáveis & $\begin{array}{c}\text { Razão de Prevalência } \\
(\text { RP) }\end{array}$ & p descritivo & $\begin{array}{c}\text { Intervalo de Confuança } \\
(\mathbf{9 5 \% )}\end{array}$ \\
\hline Sexo & & OBESIDADE & \\
Masculino & 1 & - & - \\
Feminino & 0,75 & 0,10 & {$[0,53 ; 1,06]$} \\
Idade da criança & 1,03 & 0,60 & {$[0,91 ; 1,18]$} \\
\hline \multicolumn{2}{r}{} & SOBREPESO + OBESIDADE & - \\
\hline Sexo & 1 & & {$[0,74 ; 1,08]$} \\
Masculino & 0,89 & - & {$[0,92 ; 1,07]$} \\
Feminino & 0,99 & 0,25 & \\
Idade da criança & & 0,86 &
\end{tabular}




\section{DISCUSSÃO}

A prevalência de excesso de peso (sobrepeso+obesidade) foi de $37,2 \%$ para o sexo masculino e de $33,4 \%$ para o sexo feminino. As médias de peso e altura nas diferentes idades para os sexos estão muito próximas e as médias de Índice de Massa Corporal são também próximas para os dois grupos, masculino e feminino. Verifica-se que a proporção de sobrepeso é maior do que a de obesidade, principalmente nas idades até 4 anos. As prevalências de obesidade e sobrepeso + obesidade não diferem, segundo sexo e idade das crianças.

No presente estudo, as prevalências de sobrepeso e de obesidade são elevadas e maiores que as verificadas em alguns outros estudos realizados com crianças pré-escolares.

Em Florianópolis, em pesquisa realizada por Corso et al ${ }^{25}$, com 3806 crianças menores de 6 anos de idade, residentes em áreas não carentes, foi encontrada prevalência de $6,8 \%$ de sobrepeso, sendo $3,0 \%$ em crianças na faixa etária de 2 a 6 anos e $3,8 \%$ entre as menores de 2 anos de idade.

Silva et $a l^{15}$ verificaram prevalência de sobrepeso de $22,6 \%$ e $11,3 \%$ de obesidade em 230 crianças com idades entre 2 e 5 anos, de duas escolas particulares do Recife.

Com o intuito de conhecer a saúde e nutrição de crianças menores de 5 anos que residiam em municípios de pequeno porte no Estado de São Paulo, Saldiva et al ${ }^{26}$, em amostra de 987 crianças, encontraram prevalência de obesidade de $6,6 \%$.

Neste estudo foram utilizadas como padrão de referência as curvas de crescimento recomendadas pela Organização Mundial de Saúde (2006), enquanto nos citados foi utilizado o padrão de referência National Center Health Statistics (NCHS/ CDC 2000) ${ }^{27}$. Segundo Torres et al ${ }^{28}$, as prevalências de excesso de peso, quando se utiliza o novo referencial da Organização Mundial de Saúde, são maiores do que quando se utiliza o NCHS/CDC(2000). Este fato, além das diferenças metodológicas entre os estudos, pode explicar, em parte, as diferenças entre prevalências encontradas.

Embora neste estudo não tenham sido analisadas variáveis socioeconômicas, o fato das crianças freqüentarem escolas particulares sugere que sejam crianças de famílias de melhor poder aquisitivo. Pesquisas têm encontrado prevalências de sobrepeso e obesidade maiores em crianças e adolescentes de famílias pertencentes a esse grupo socioeconômico quando comparado com grupos de menor poder

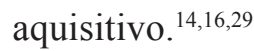

Monteiro \& Conde,,$^{10} \mathrm{em}$ pesquisa sobre tendência secular da desnutrição e da obesidade na infância na Cidade de São Paulo (19741996), verificaram, que naquele período, a desnutrição deixou de ser endêmica na cidade, enquanto que o risco de obesidade era restrito às crianças pertencentes às famílias mais ricas, na faixa etária de 0 a 59 meses.

Em estudo recente com 3957 crianças com idades entre 1 mês e 5 anos, foi encontrada prevalência de excesso de peso de $9,8 \%$, sendo um dos fatores associados à chance de excesso de peso o elevado nível socioeconômico, ${ }^{17} \mathrm{o}$ que parece indicar que, pelo menos na infância, pertencer a família de nível social elevado aumenta o riso de excesso de peso.

A obesidade infantil, segundo a Organização Mundial de Saúde, tem crescido em torno de 10 a $40 \%$ na maioria dos países europeus, nos últimos 10 anos, ocorrendo com mais freqüência no primeiro ano de vida, entre 5 e 6 anos e na adolescência. ${ }^{30}$

Neste estudo, quando se considera a prevalência de obesidade, ou de sobrepeso + obesidade não é encontrada diferença significativa entre os sexos e à medida que a idade avança. Isto indica, e a Figura 1 mostra, que na faixa etária de 2 a 6 anos, o processo de estabelecimento de alterações de peso é estável, podendo sofrer alterações mais à frente, em crianças em idade escolar e na adolescência. 
Balaban \& Silva ${ }^{29}$ encontraram, em crianças de 6 a 9 anos, diferença significativa entre as prevalências de obesidade segundo sexo, sendo a maior prevalência verificada entre os meninos.

A obesidade infantil já pode ser considerada um problema de saúde pública, que atinge precocemente crianças, tornando-se sério agravo para a saúde atual e futura dos indivíduos. Prevenir a obesidade infantil significa diminuir de forma racional e menos onerosa a incidência de doenças crônico-degenerativas na vida adulta. ${ }^{11}$

\section{REFERÊNCIAS}

1. De Angelis RC. Riscos e prevenção da obesidade: fundamentos fisiológicos e nutricionais para o tratamento. Atheneu: São Paulo, 2003.

2. Parra- Caberá S, Hernández B, DuranArenas L, López-Arellano O. Modelos alternativos para el análisis epidemiológico de la obesidad como problema de salud pública. Rev. Saúde Pública. 1999;33:314-25.

3. Pérusse L, Bouchard C. Gene diet interactions in obesity. Am J Clin Nutr.2000;72 Supp15:S1285-90.

4. Troiano RP, Briefel RR, Carrol MD, Bialostosky K. Energy and fat intakes of children and adolescents in the United States: data from the National Health and Nutrition Examination Surveys. Am J Clin Nutr. 2000;72 Suppl 5:S1343-53.

5. Atkin LM, Davies PSW. Diet composition and body composition in preschool children. Am J Clin Nutr.2000;72:15-21.

6. Lustig RH. The neuroendocrinology of childhood obesity. Pediatr Clin North Am. 2001;48:1-14.

7. Meyer F, Mello ED, Luft VC. Obesidade infantil: como podemos ser eficazes? J Pediatr. 2004;80(3):173-82.
Assim, os resultados do presente estudo mostram elevada prevalência de excesso de peso em crianças de escolas particulares do município de São Paulo, independente da idade e do sexo da criança. Acredita-se que são indispensáveis programas de prevenção da obesidade infantil. A avaliação nutricional e programas de reeducação alimentar deveriam ser fortes aliados contra a obesidade em escolas, consultórios de pediatria, e serviços públicos de saúde, de modo a tentar controlar os problemas de excesso de peso, principalmente em idades mais precoces, como a dos pré-escolares.

8. World Health Organization. WHO Obesity - Preventing and managing the global epidemic. Report of WHO Consutation on Obesity. Geneva.1997; 7-16.

9. Martorell R. Khan LK, Hughes ML, Grummer-Strawn LM. Overweight and obesity in preschool children from developing countries. Int J Obes 2000:24:959-67.

10. Monteiro CA, Conde WL. Tendência secular da desnutrição e da obesidade na infância na cidade de São Paulo (19741996). Rev. Saúde Pública 2000; 34(6 Supl):52-61.

11. Abrantes MM, Lamounier JÁ, Colosimo EA. Prevalência de sobrepeso e obesidade em crianças e adolescentes das Regiões Sudeste e Nordeste do Brasil. J Pediatr. 2002; 78(4):335-40.

12. Chinn S, Rona RJ. Prevalence and trends in overweight and obesity in three crosssectional studies of British children.

BMJ. 2001; 322(7277):24-6.

13. Magalhães VC, Mendonça GAS. Prevalência e fatores associados a sobrepeso e obesidade em adolescentes de 15 a 19 anos das regiões Nordeste e Sudeste do Brasil, 1996 a 1997. Cad Saúde Pública. 2003; 19 (Sup 1):129-39.

14. Leão LSCS, Araújo LMB, Moraes LTLP, Assis AM. Prevalência de obesidade em 
escolares de Salvador, Bahia. Arq Bras Endocrinol Metab. 2003; 47(2):151-7.

15. Silva GAP, Balaban G, Freias MMV, Baracho JDS, Nascimento EMM. Prevalência de sobrepeso e obesidade em crianças pré-escolares matriculadas em duas escolas particulares de Recife, Pernambuco. Rev Bras. Saúde Matern. Infant. 2003; 3(3)323-27.

16. Ronque ERV, Cyrino ES, Dorea VR, Junior HS, Galdi EHG, Arruda M. Prevalência de sobrepeso e obesidade em escolares de alto nível socioeconômico em Londrina, Paraná, Brasil. Rev Nutr. 2005;18(6):709-717.

17. Vitolo MR, Gama CM, Bortolini GA, Campagnolo PD, Drachler ML. Some risk factors associated with overweight, stunting and wasting among children under 5 years old. J Pediatr (Rio J).2008; 84(3):251-257.

18. Malina RM, Bouchard C, Bar-Or O. Growth, maturation, and physical activity. $2^{\text {nd }}$ ed. Champaign: Human Kinetics Books; 2004.

19. Instituto Nacional de Alimentação e Nutrição. Pesquisa Nacional sobre Saúde e Nutrição- PNSN- 1989. Brasília; 1990 [Arquivos de dados de pesquisa].

20. Ministério da Saúde. Política Nacional de Demografia e Saúde. Saúde e estado nutricional de crianças menores de 5 anos. Brasil, 2006. [citado 13.08.2008]. Disponível http://bvsms.saude.gov.br/ bvs/pnds/saude_nutricional.php.

21. Monteiro CA, Benicio D'Aquino MH, Ortiz LP. Tendência secular do peso ao nascer na cidade de São Paulo (19761998).Rev Saúde Pública. 2000;34(6Supl):26-40.

22. Lohman TG, Roche AF, Matorell R. Antropometric standardization reference manual.1988. Iiinois: Human Kinetics Publishers.

23. Onis M, Garza C, Onyango A, Borghi E. Comparison of the WHO child growth standards and the CDC 2000 growth charts. J Nut. 2007; 137:144-148.

24. Harden J e Hilbe J. The general binomial family. In: Harden J \& Hilbe J. Generalized linear models and extensions. Texas:College Station:Statapress;2001.

25. Corso ACT, Botelho LJ, ZeniI Larz, Moreira EAM. Sobrepeso em crianças menores de 6 anos de idade em Florianópolis. Rev Nutr. 2003; 16(1)21-28.

26. Saldiva SRDM, Escuder MML, Venâncio SI, Benicio D’ Aquino MH.Prevalence of obesity in preschool children from five towns in Sao Paulo state, Brazil. Cad. Saúde Pública. 2004;20(6): 1627-32.

27. Kuczmarski RJ, Ogden CL, Grummer SLM et al. CDC Growth Charts: United States - methods and developmente. National Center for Health Statistics. Vital Health Statistics.2000:246.

28. Torres AAL, Furumoto RAV, Alves ED. Avaliação antropométrica de préescolares - comparação entre referências: NCHS 2000 e OMS 2005. Revista Eletrônica de Enfermagem. 2007; 9(1): 166-75 [Disponível em http:/ /www.fen.ufg.br/revista/v9/n1/ v9n1a13.htm

29. Balaban G e Silva GAP. Prevalência de sobrepeso e obesidade em crianças e adolescentes de uma escola da rede privada de Recife. J Pediatr (Rio J).2001; 77(2): 96-100.

30. Ebbeling CB, Pawlak DB, Ludwig DS. Childhood obesity: public-health crisis, common sense cure. Lancet. 2002; 360(9331):473-82.

Recebido em 28 de março de 2009. Modificado em 10 de julho de 2009 Aceito em 26 de agosto de 2009 